\title{
Equilibrium electrons in free-electron lasers with a 3D helical wiggler and a guide magnetic field: Nonlinear simulations
}

\author{
Xiao-Li Huang, ${ }^{1}$ Shi-Jian Wang, ${ }^{2}$ Yong-Gen Xu, ${ }^{2}$ and Shi-Chang Zhang ${ }^{1,3, *}$ \\ ${ }^{1}$ School of Electrical and Information Engineering, Xihua University, Chengdu 610039, China \\ ${ }^{2}$ School of Physics and Chemistry, Xihua University, Chengdu 610039, China \\ ${ }^{3}$ Institute of Photoelectronics, Campus Mail Box 50, School of Information Science and Technology, \\ Southwest Jiaotong University, Chengdu 610031, China \\ (Received 2 June 2012; published 17 December 2012)
}

\begin{abstract}
Nonlinear simulations are devoted to the comparative study of the equilibrium electrons' motion and stability in the combination of a three-dimensional helical wiggler and a positive or reversed guide magnetic field, where effects of the self-field, off-axis guiding center and adiabatic magnetic field are included. It is shown that a reversed guide magnetic field configuration brings the electron motion much smaller Larmor radius and transverse displacement span than a positive guide magnetic field does, and consequently, provides better transportation quality of the electron beam. Although the electron motion far from the resonance and antiresonance is stable in both the positive and the reversed guide magnetic field configurations, fluctuation induced by a reversed guide magnetic field is smaller than that by a positive guide magnetic field, especially in the adiabatic region of the wiggler. In a certain parameters domain, the self-field of the electron beam is advantageous to confine the electrons' motion at antiresonance.
\end{abstract}

DOI: 10.1103/PhysRevSTAB.15.120702

PACS numbers: 41.60.Cr, 52.59.Rz

\section{INTRODUCTION}

Free-electron lasers (FELs) with the combination of a wiggler and a positive or a reversed guide magnetic field have been recognized as a kind of high-power coherent radiation sources in the infrared, terahertz, and millimeter wave ranges, and have continuously drawn wide attention [1-25]. Use of a positive or a reversed guide magnetic field brings to this kind of FELs advantages such as gain enhancement and the electron-beam confinement [1-4]. As demonstrated in the first paper of this series [25], on the other hand, these two guide magnetic fields have unnegligible differences in the functions on the equilibrium electrons' motion and stability.

By means of nonlinear simulations, this paper presents a comparative investigation for the functions of a positive guide magnetic field and a reversed guide magnetic field on the equilibrium electrons' motion, trajectory stability, and the electron-beam transmission. Although previous perturbation analysis and Poincaré-map analysis have been devoted to this subject, fundamental approximation was presupposed in these analytical derivations; for example, either the wiggler field was restricted to have onedimensional distribution $[3,4,9,11,12]$ or was averaged over the cyclotron angle [8], or the electron's guiding center was

\footnotetext{
*Corresponding author. sczhang@home.swjtu.edu.cn
}

Published by the American Physical Society under the terms of the Creative Commons Attribution 3.0 License. Further distribution of this work must maintain attribution to the author(s) and the published article's title, journal citation, and DOI. treated to be on axis [5-7,10], and the adiabatic field over the initial region of the wiggler was mostly neglected. In practice, however, these factors may substantially affect the equilibrium electrons' motion, stability, and transmission. To approach the practical situations and pursue more precise description, we shall fully consider these factors in our model of the nonlinear simulations.

We organize this paper as follows. In Sec. II a basic model of the nonlinear simulations is developed, where the adiabatic and three-dimensional distributions of a helical wiggler field, the self-field, and the off-axis guiding centers of the electron beam are taken into account. Then, in Sec. III, in terms of the experimental parameters reported by Conde and Bekefi [13], comparative simulations are carried out for the functions of a positive guide magnetic field and a reversed guide magnetic field. In Sec. IV, by means of the Kolmogorov entropylike quantity, the stability of the equilibrium electrons' motion is studied for the positive and reversed guide magnetic fields and off-axis guiding centers. Specific discussion is presented for the situations at resonance and at antiresonance in Sec. V. Finally, conclusions are drawn in Sec. VI.

\section{NONLINEAR SIMULATION MODEL}

In this section we establish a basic model of the nonlinear simulations for the equilibrium electrons' motion and the electron-beam transport. Suppose that an equilibrium electron beam immersed in a $3 \mathrm{D}$ wiggler magnetic field $\vec{B}_{w}$ and an axial guide magnetic field $\vec{B}_{0}$ drafts in a cylindrical waveguide with radius $R_{w}$. An arbitrary test electron is governed by the relativistic Lorentz equation 


$$
\frac{d\left(\gamma m_{0} \vec{v}\right)}{d t}=-e\left[\vec{E}_{b}+\vec{v} \times\left(\vec{B}_{w}+\vec{B}_{0}+\vec{B}_{b}\right)\right]
$$

with

$$
\gamma=\left(1-v^{2} / c^{2}\right)^{-1 / 2},
$$

where $m_{0}, e, \vec{v}$, and $\gamma$ are the electron's rest mass, charge, velocity, and Lorentz energy factor, respectively. In the above equations, $\vec{B}_{w}$ is the magnetic field of a threedimensional helical wiggler with amplitude $B_{A}$ and wave number $k_{w}$, which can be expressed as [5]

$$
\begin{aligned}
\vec{B}_{w}= & 2 B_{A} I_{1}^{\prime}\left(k_{w} R\right) \cos \left(\varphi-k_{w} R\right) \hat{e}_{R}-2 B_{A} \frac{I_{1}\left(k_{w} R\right)}{k_{w} R} \\
& \times \sin \left(\varphi-k_{w} R\right) \hat{e}_{\varphi}+2 B_{A} I_{1}\left(k_{w} R\right) \sin \left(\varphi-k_{w} R\right) \hat{e}_{z},
\end{aligned}
$$

$\vec{B}_{0}$ is the axial guide magnetic field with a constant magnitude $B_{0}$ :

$$
\vec{B}_{0}=B_{0} \hat{e}_{z}
$$

$\vec{B}_{b}$ and $\vec{E}_{b}$ are the magnetic and the electric components of the electron-beam self-fields [26]:

$$
\begin{gathered}
\vec{E}_{b}= \begin{cases}-\frac{e n_{b}}{2 \varepsilon_{0}} R \hat{\mathrm{e}}_{R}, & R<R_{b} \\
-\frac{e n_{b}}{2 \varepsilon_{0}} \frac{R_{b}^{2}}{R} \hat{\mathrm{e}}_{R}, & R \geq R_{b},\end{cases} \\
\vec{B}_{b}= \begin{cases}-\frac{e \mu_{0} n_{b} v_{z 0}}{2} R \hat{\mathrm{e}}_{\varphi}, & R<R_{b} \\
-\frac{e \mu_{0} n_{b} v_{z 0}}{2} \frac{R_{b}^{2}}{R} \hat{\mathrm{e}}_{\varphi}, & R \geq R_{b},\end{cases}
\end{gathered}
$$

where $k_{w}=2 \pi / \lambda_{w}, \lambda_{w}$ is the wiggler period, $R_{b}, n_{b}$, and $v_{z 0}$ are the electron-beam radius, volume density, and axial average speed, $I_{l}\left(k_{w} R\right)$ and $I_{l}^{\prime}\left(k_{w} R\right)$ are the first kind of modified Bessel functions of order $l$ and its derivative with respect to the whole argument, $\hat{e}_{R}, \hat{e}_{\varphi}$, and $\hat{e}_{z}$ are the unit vectors in the radial, azimuthal, and axial directions in a cylindrical coordinate system $(R, \varphi, z)$, respectively. Here $B_{0}>0$ denotes a positive guide magnetic field while $B_{0}<0$ denotes a reversed guide magnetic field.

It should be emphasized that, to take the adiabatic region of the wiggler into account, we treat the wiggler amplitude $B_{A}$ as a function of the axial position $z$ :

$$
B_{A}= \begin{cases}B_{w} f(z), & z<N_{w} \lambda_{w} \\ B_{w}, & z \geq N_{w} \lambda_{w},\end{cases}
$$

where $B_{w}=$ const is the magnitude of the wiggler amplitude, $N_{w}$ and $f(z)$ are the period number and the field intensity function in the adiabatic region of the wiggler. The measured profile of the wiggler field as a function of the axial position in the MIT experimental setup was reported by Fajans et al. (see Fig. 4 in Ref. [24]), and simulating that measured profile, we choose

$$
\begin{aligned}
f(z)= & 0.01702514-0.0007826626\left(k_{w} z\right) \\
& +0.000734124\left(k_{w} z\right)^{2}-0.0000004168\left(k_{w} z\right)^{3},
\end{aligned}
$$

when we employ the MIT experimental parameters in our simulations. Elsewhere, we choose the following function [27]:

$$
f(z)=\left(\frac{\sin \bar{z}}{4 N_{w}}\right)^{2} .
$$

To reduce the accumulated iteration error in the nonlinear simulations, we simplify the right-hand functions of Eq. (1) by introducing $\vec{V}=\gamma \vec{v}$, and then rewrite it as six scalar equations of the velocity variables and the configuration variables [15]:

$$
\begin{gathered}
\frac{d \bar{V}_{x}}{d \bar{z}}=-\frac{1}{\bar{V}_{z}}\left[\bar{E}_{x} \sqrt{1+\bar{V}^{2}}+\left(\bar{V}_{y} \bar{B}_{z}-\bar{V}_{z} \bar{B}_{y}\right)\right], \\
\frac{d \bar{V}_{y}}{d \bar{z}}=-\frac{1}{\bar{V}_{z}}\left[\bar{E}_{y} \sqrt{1+\bar{V}^{2}}+\left(\bar{V}_{z} \bar{B}_{x}-\bar{V}_{x} \bar{B}_{z}\right)\right], \\
\frac{d \bar{V}_{z}}{d \bar{z}}=-\frac{1}{\bar{V}_{z}}\left[\bar{E}_{z} \sqrt{1+\bar{V}^{2}}+\left(\bar{V}_{x} \bar{B}_{y}-\bar{V}_{y} \bar{B}_{x}\right)\right], \\
\frac{d \bar{x}}{d \bar{z}}=\frac{\bar{V}_{x}}{\bar{V}_{z},} \\
\frac{d \bar{y}}{d \bar{z}}=\frac{\bar{V}_{y}}{\bar{V}_{z}}, \\
\frac{d \bar{t}}{d \bar{z}}=\frac{\sqrt{1+\bar{V}^{2}}}{\bar{V}_{z}},
\end{gathered}
$$

where $\bar{x}=k_{w} x, \bar{y}=k_{w} y, \bar{z}=k_{w} z, \bar{t}=c k_{w} t, \bar{V}=\gamma v / c$, $\bar{B}=|e| B /\left(m_{0} c k_{w}\right)$, and $\bar{E}=|e| E /\left(m_{0} c^{2} k_{w}\right)$ are the dimensionless quantities, $c$ is the light speed in vacuum, and transformation of $\frac{d}{d t}=\frac{d z}{d t} \frac{d}{d z}$ has been made.

In the simulations we employ the Runge-Kutta method of order four, and use 5000 test electrons with the initial states of Gaussian random distribution in the velocity space and configuration space to represent the electron beam. In programming the code, two techniques are adopted to ensure the reliability. First, if a test electron strikes the waveguide wall before the exit of the wiggler, the electronbeam volume density $n_{b}$ in Eqs. (5) and (6) will be automatically reduced in proportion to the lost number over the initial number of the test electrons. Second, the iteration step is chosen to be at the order of $10^{-7}$ of the wiggler period so as to reflect the precise influence of the wiggler on the test electrons' motion. 


\section{ELECTRON MOTION AND BEAM TRANSPORT}

We compare the functions of the positive guide magnetic field and the reversed guide magnetic field by employing the parameters in the experiments conducted at the Massachusetts Institute of Technology (MIT) [13]. In the MIT experiment the setup had the basic parameters of a wiggler period $\lambda_{w}=3.18 \mathrm{~cm}$, a wiggler length $L_{w}=$ $200 \mathrm{~cm}$, an average beam energy factor $\gamma_{0}=2.4677$, a beam radius $R_{b}=0.25 \mathrm{~cm}$, and a waveguide radius $R_{w}=0.52 \mathrm{~cm}$.

Figure 1 shows the cross-sectional view of a testelectron motion evolution and the electron-beam spot at the exit in the case of a reversed guide magnetic field $B_{0}=-10900 \mathrm{G}$, where the wiggler magnitude is $1470 \mathrm{G}$, the test electron has the initial conditions $\left.x\right|_{t=0}=$ $0.127 \mathrm{~cm},\left.y\right|_{t=0}=0,\left.z\right|_{t=0}=0,\left.v_{x}\right|_{t=0}=0,\left.v_{y}\right|_{t=0}=0$, and $\left.v_{z}\right|_{t=0}=c \sqrt{\gamma_{0}^{2}-1} / \gamma_{0}$, and the electron beam has an initial current of $300 \mathrm{~A}$ and an energy spread of $1 \%$. In order to compare the different effects between a positive guide field and a reversed guide field, we plot Fig. 2 by using a positive guide field $B_{0}=10900 \mathrm{G}$ instead of the reversed guide field $B_{0}=-10900 \mathrm{G}$ in Fig. 1 .

Figures 1 and 2 show a property common to the reversed guide magnetic field and the positive guide magnetic fields: after passing through the adiabatic region of the wiggler, approximately, the electron's rotation has an invariant Larmor radius, and its guiding center shifts with an invariant angular velocity and a constant radial position. Meanwhile, a different property can be found in the figures: the positive guide magnetic field leads the test electron to have much greater Larmor radius and larger transverse velocity and to span a wider range in velocity and configuration than the reversed guide magnetic field does. These nonlinear results coincide with that predicated by the explicit formulation in the first paper of this series [25]. Here one may conclude that a reversed guide magnetic field can provide a better transportation quality for the electron beam with smaller velocity spread and configuration spread than a positive guide magnetic field. As confirmed by Figs. 1(d) and 2(d), the spot of the (a)

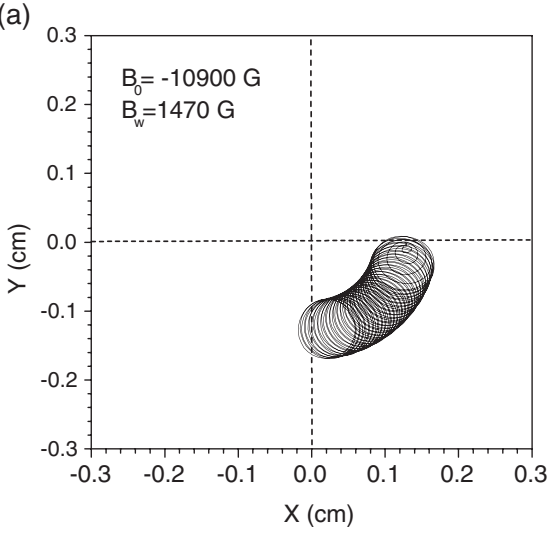

(c)

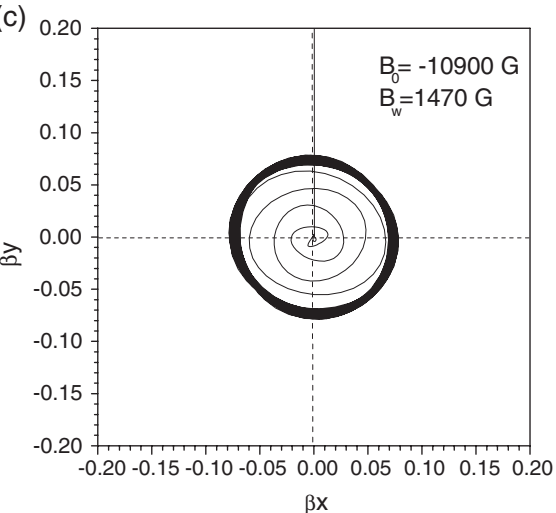

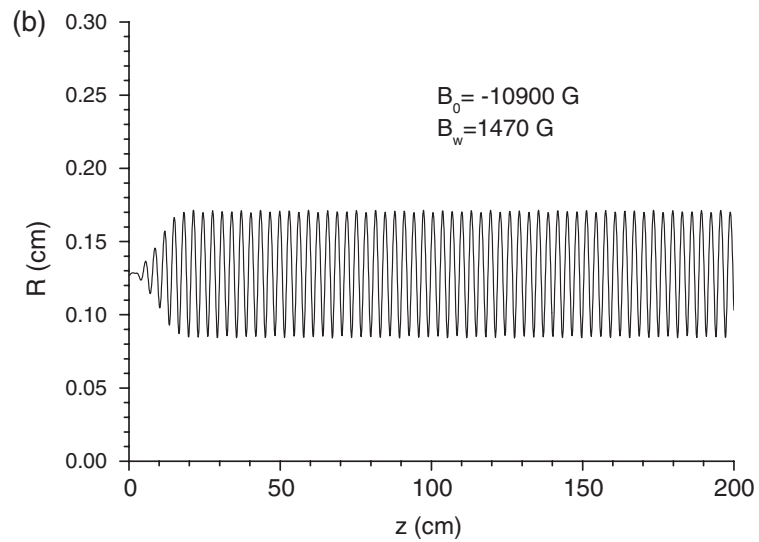

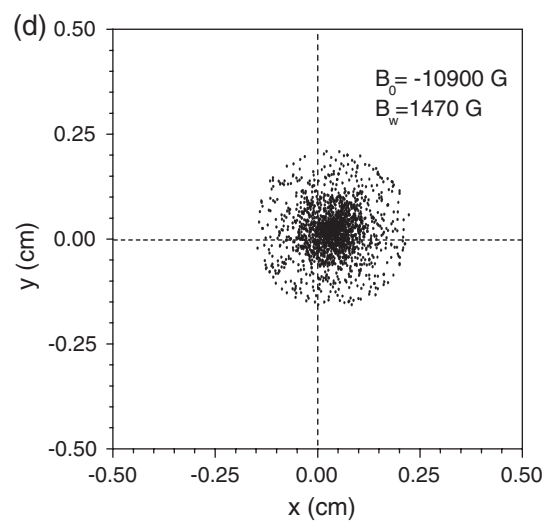

FIG. 1. Cross-sectional view of a test electron's motion evolution (a), (b), (c), and the electron-beam spot at the exit (d) in a reversed guide magnetic field $B_{0}=-10900 \mathrm{G}$, where the parameters are from the MIT experiment [13]: the wiggler has amplitude $B_{w}=$ $1470 \mathrm{G}$, period $\lambda_{w}=3.18 \mathrm{~cm}$, adiabatic period number $N_{w}=6$, and length $L_{w}=200 \mathrm{~cm}$; the test electron has the initial conditions $\left.x\right|_{t=0}=0.127 \mathrm{~cm},\left.y\right|_{t=0}=0,\left.z\right|_{t=0}=0,\left.v_{x}\right|_{t=0}=0,\left.v_{y}\right|_{t=0}=0$, and $\left.v_{z}\right|_{t=0}=c \sqrt{\gamma_{0}^{2}-1} / \gamma_{0}$; the electron beam has initial current $I_{b}=300 \mathrm{~A}$, energy factor $\gamma_{0}=2.4677$, energy spread $\Delta \gamma / \gamma_{0}=1 \%$, and beam radius $R_{b}=0.25 \mathrm{~cm}$; the waveguide has radius $R_{w}=0.52 \mathrm{~cm}$. Here in diagram (d) only 2000 of the 5000 test electrons are plotted due to the publication file size limited. 

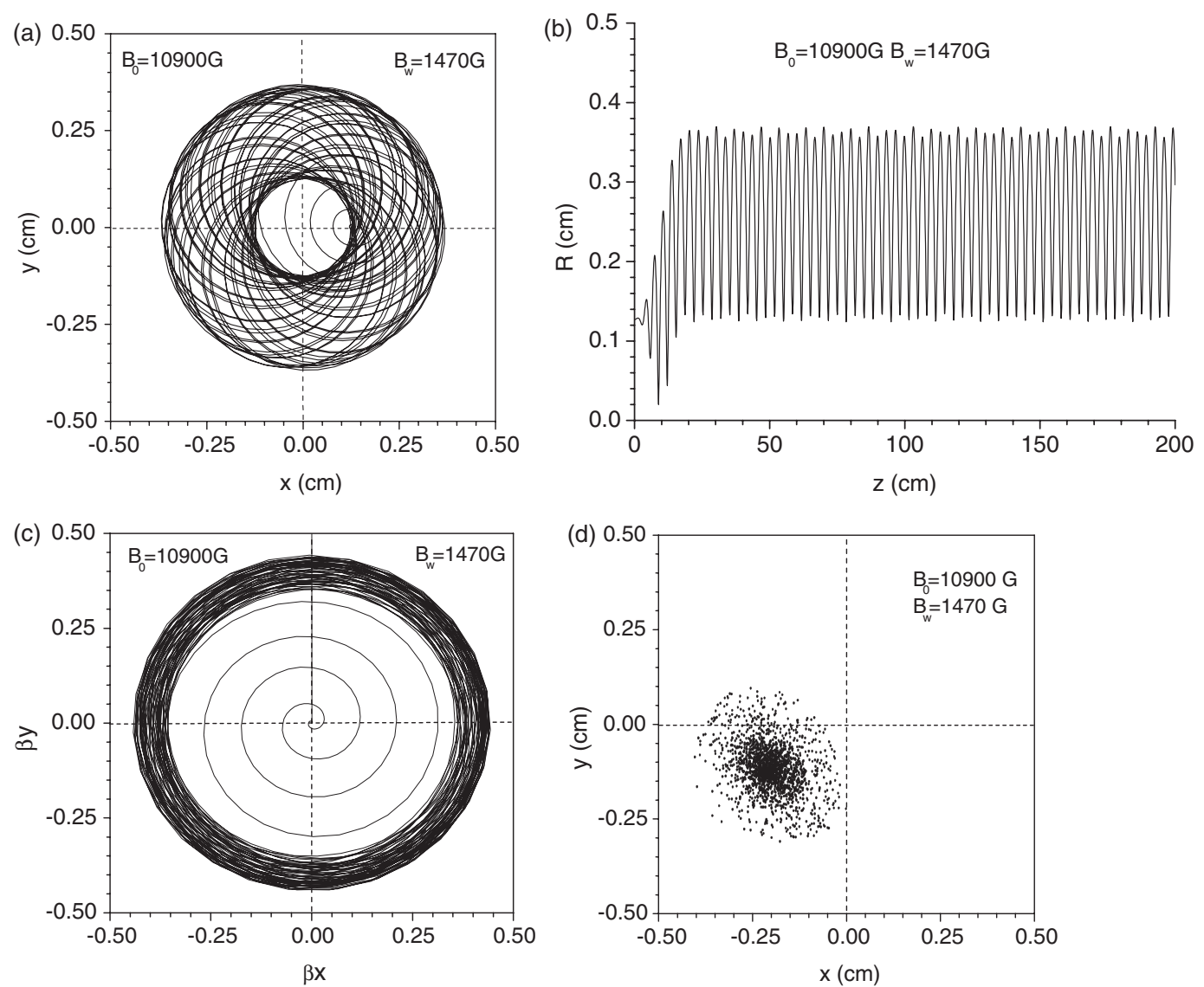

FIG. 2. Cross-sectional view of a test electron's motion evolution (a), (b), (c), and the electron-beam spot at the exit (d) in a positive guide magnetic field $B_{0}=10900 \mathrm{G}$, where the other parameters are the same as in Fig. 1. Here in diagram (d) only 2000 of the 5000 test electrons are plotted due to the publication file size limited.

electron beam at the exit of the wiggler in the case of a positive guide magnetic field gets harmful distortion and serious deviation from the axis, whereas the electron-beam spot at the exit in the case of a reversed guide magnetic field retains a perfect state.

\section{STABILITY OF ELECTRON MOTION}

As performance of a FEL is sensitive to multiparameters, stability of the electrons' motion is of importance to a FEL operation. In the past years, stability of the equilibrium electrons' motion in a FEL with helical wiggler and axial guide magnetic field has been intensively studied in the perturbation analysis and the Poincaré-map analysis (for example, see Refs. [6-12]). However, it is by no means definitive. Because of the complexity in mathematical derivation, these analytical methods are hardly used to treat the electron's motion in the adiabatic region of a 3D helical wiggler, and the effect of the adiabatic field on the motion stability was always neglected.

Benettin et al. [28] developed an effective approach to estimate the rate of divergence of two nearby particles' phase-space trajectories by computing the entropylike quantity $k$, which was derived from the Kolmogorov entropy:

$$
k=\lim _{\substack{l \rightarrow \infty \\\left|d_{0}\right| \rightarrow 0}}\left(\frac{1}{t}\right) \ln \left(\frac{|d(t)|}{\left|d_{0}\right|}\right),
$$

where the norm $|d(t)|$ is defined as the six-dimensional phase-space distance between these two nearby particles,

$$
|d(t)|=\sqrt{\sum_{j=1}^{6}\left(x_{j n}-x_{j r}\right)^{2}} .
$$

Kolmogorov entropylike quantity $k$ measures the sensitivity of the particle's motion to the initial conditions: if $|d(t)|$ grows exponentially with time $t$, i.e., $|d(t)| \propto$ $\exp (\lambda t)$, these two nearby particles' trajectories will exponentially diverge for positive $\lambda(>0)$ and chaos may occur, whereas the trajectories will exponentially converge for negative $\lambda(<0)$; the smaller $k$ is, the more stable is the electron's motion. By solving the velocity and configuration equations (10)-(15), two nearby equilibrium electrons' phase-space trajectories and the Kolmogorov entropylike quantity $k$ can be easily traced, no matter 

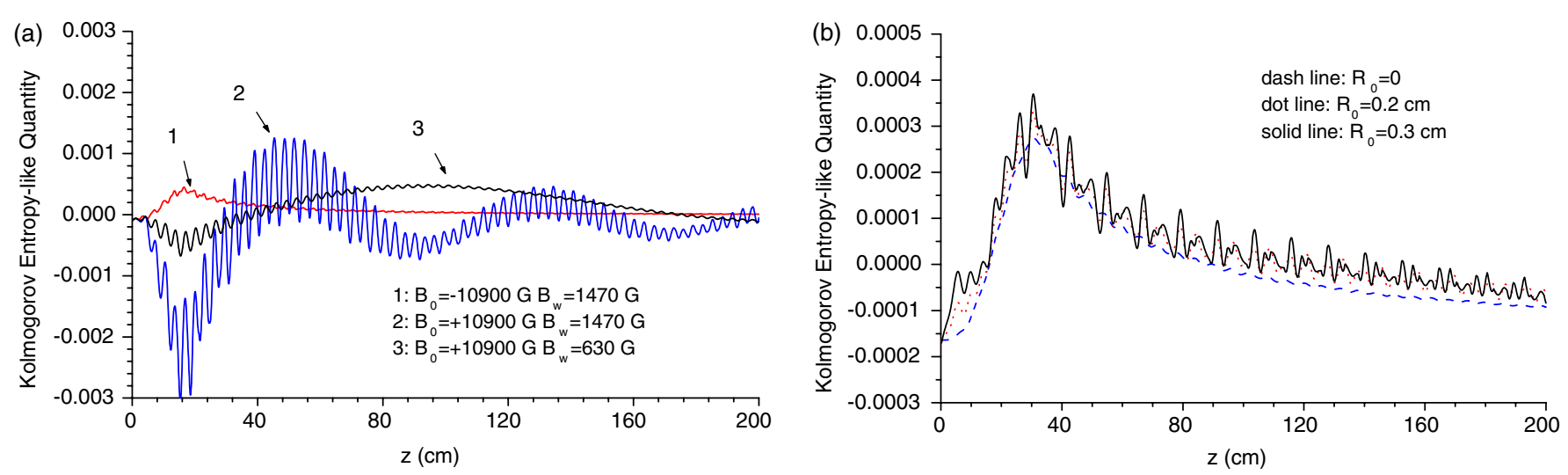

FIG. 3. Kolmogorov entropylike quantity of a test electron's motion: (a) in different guide magnetic field configurations for the parameters in the MIT experiment [13] shown in Fig. 1; (b) in a reversed guide magnetic field configuration for initial on-axis and off-axis conditions, where the parameters are from the JINR experiment [21]: the wiggler has period $\lambda_{w}=6 \mathrm{~cm}$, amplitude $B_{w}=1000 \mathrm{G}$, length $L_{w}=216 \mathrm{~cm}$, and adiabatic period number $N_{w}=6$; the electron beam has current $I_{b}=200$ A, energy factor $\gamma_{0}=2.6$, energy spread $\Delta \gamma / \gamma_{0}=1 \%$, and beam radius $R_{b}=0.4 \mathrm{~cm}$; the waveguide has radius $R_{w}=0.95 \mathrm{~cm}$.

how complex the magnetic or electric field that is applied to the electrons.

Figure 3(a) plots the comparison of the Kolmogorov entropylike quantity for the positive guide magnetic field and the reversed guide magnetic field by use of the MIT experiment parameters [13]. To ensure the reliability of the simulation results, we choose the iteration step to be $2 \times 10^{-7} \mathrm{~cm}$, which corresponds to a total iteration number of $10^{9}$. It displays that no chaos of the electrons' dynamic behavior occurs for both the positive guide magnetic field and the reversed guide magnetic field, and the test electrons' motion tends toward stableness. Nevertheless, it evidently indicates that the electron motion in a positive guide magnetic field gets a greater fluctuation than that in a reversed guide magnetic field, especially in the adiabatic region of the 3D helical wiggler.

Figure 3(b) presents a comparison of the motion stability of the test electrons with initial on-axis and off-axis guiding centers in the case of a reversed guide magnetic field, where the parameters are from the JINR experiment [21]: the wiggler had a period $\lambda_{w}=6 \mathrm{~cm}$, a field magnitude $B_{w}=1000 \mathrm{G}$, a length $L_{w}=216 \mathrm{~cm}$, and an adiabatic period number $N_{w}=6$; the electron beam had an average energy factor $\gamma_{0}=2.6$, a current $I_{b}=200 \mathrm{~A}$, and a radius $R_{b}=0.4 \mathrm{~cm}$; the waveguide had a radius $R_{w}=0.95 \mathrm{~cm}$. It is clear from the figure that after passing through the adiabatic region of the wiggler, the test electrons' motion is stable and motion fluctuation is small, no matter if the initial guiding center is on axis or off axis. It confirms that use of a reversed guide magnetic field is able to provide a stable electron-beam transport.

\section{RESONANCE AND ANTIRESONANCE}

One of the focuses on the equilibrium electrons' motion in a FEL with a guide magnetic field is the states at resonance and at antiresonance. In this section we first discuss the resonance in a positive guide magnetic field configuration, then the antiresonance in a reversed guide magnetic field configuration.

In a positive guide magnetic field configuration, the early perturbation analysis for a one-dimensional (1D) wiggler gave the necessary and sufficient condition of an equilibrium electron's Larmor orbit [4]:

$$
v_{\perp 0}=\frac{ \pm 2 v_{\| 0} \Omega_{w}}{k_{w} v_{\| 0}-\Omega_{0}}
$$

where $\Omega_{0}=\frac{e B_{0}}{\gamma m_{0}}$ and $\Omega_{w}=\frac{e B_{w}}{\gamma m_{0}}$. Later, it was extended to a three-dimensional (3D) helical wiggler for the electrons with on-axis guiding centers [5,6]:

$$
v_{\perp 0}=\frac{ \pm 2 v_{\| 0} \Omega_{w} I_{1}\left(\bar{r}_{L}\right) / \bar{r}_{L}}{k_{w} v_{\| 0}-\Omega_{0} \mp 2 \Omega_{w} I_{1}\left(\bar{r}_{L}\right)} .
$$

In the first paper of this series [25], we generalized it to the electrons with off-axis guiding centers,

$$
v_{\perp 0}=\frac{ \pm 2 v_{\| 0} \Omega_{w} I_{0}\left(\bar{R}_{g}\right) I_{1}\left(\bar{r}_{L}\right) / \bar{r}_{L}}{k_{w} v_{\| 0}-\Omega_{0} \mp 2 \Omega_{w} I_{0}\left(\bar{R}_{g}\right) I_{1}\left(\bar{r}_{L}\right)},
$$

deduced the formula of the Larmor radius,

$$
\bar{r}_{L}^{2}=\frac{ \pm 2 \Omega_{w} I_{0}\left(\bar{R}_{g}\right) I_{1}\left(\bar{r}_{L}\right)}{k_{w} v_{\| 0}-\Omega_{0} \bar{\mp} 2 \Omega_{w} I_{0}\left(\bar{R}_{g}\right) I_{1}\left(\bar{r}_{L}\right)},
$$

and revealed the Larmor-radius resonance at $\Omega_{0}=$ $k_{w} v_{\| 0} \mp 2 \Omega_{w} I_{0}\left(\bar{R}_{g}\right) I_{1}\left(\bar{r}_{L}\right)$.

There is a property common to Eqs. (18)-(21): limit of the transverse velocity resonance at the singularity is infinite, which causes strike of the equilibrium electron on the waveguide wall before the exit of the wiggler. On the other hand, there exists a substantial difference among these equations: Eq. (18) for a 1D wiggler implies that the 

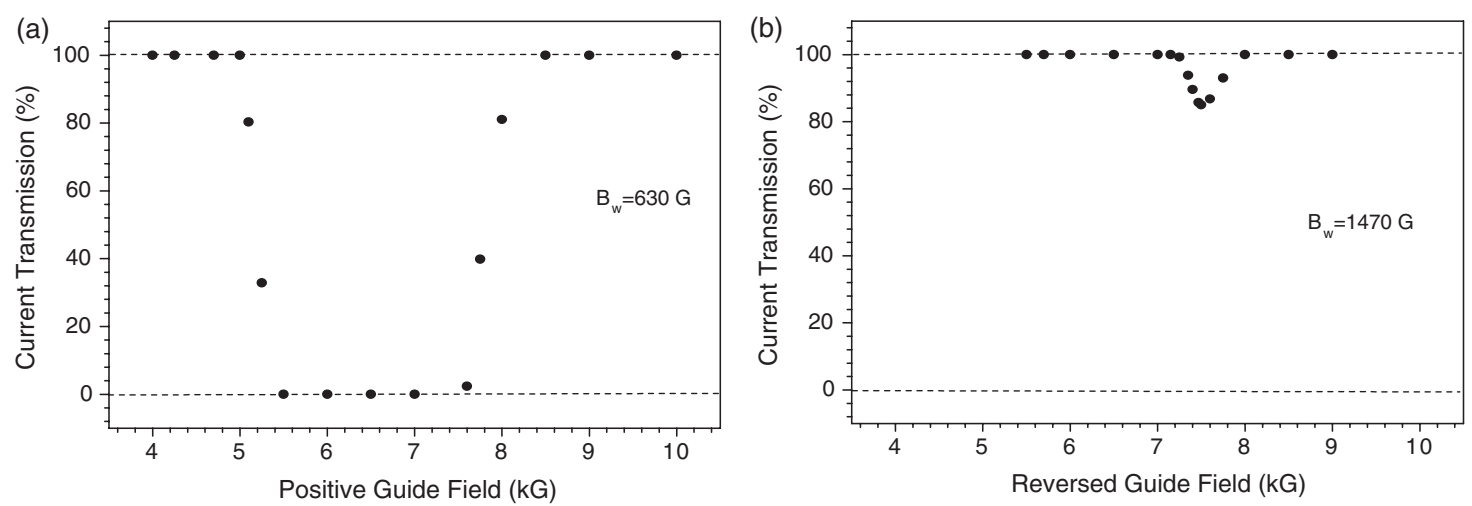

FIG. 4. Beam current transmission as a function of a positive guide magnetic field (a), and a reversed guide magnetic field (b), where the other parameters are the same as in Fig. 1.

transverse velocity resonance or strike of the electron on the waveguide wall occurs when the positive guide magnetic field is at the point $\Omega_{0}=k_{w} v_{\| 0}$, whereas Eqs. (19) and (20) for a 3D wiggler indicates that it occurs in a range $\Omega_{0}=k_{w} v_{\| 0} \mp 2 \Omega_{w} I_{1}\left(\bar{r}_{L}\right)$ for the on-axis guiding centers, or more generally, in a range $\Omega_{0}=k_{w} v_{\| 0} \mp$ $2 \Omega_{w} I_{0}\left(\bar{R}_{g}\right) I_{1}\left(\bar{r}_{L}\right)$ for both the on-axis and the off-axis guiding centers. In other words, even if $\Omega_{0} \neq k_{w} v_{\| 0}$, resonance can occur by alternating $\bar{r}_{L}$ or $\bar{R}_{g}$ to satisfy the resonance condition $\Omega_{0}=k_{w} v_{\| 0} \mp 2 \Omega_{w} I_{1}\left(\bar{r}_{L}\right)$ for the on-axis electrons or $\Omega_{0}=k_{w} v_{\| 0} \mp 2 \Omega_{w} I_{0}\left(\bar{R}_{g}\right) I_{1}\left(\bar{r}_{L}\right)$ for the off-axis electrons.

Based on the nonlinear model in Sec. II, beam current transmission as a function of the positive guide magnetic field is plotted in Fig. 4(a) by using the parameters of the MIT experiment [13], where the beam current transmission is defined as the ratio of the current at the exit to that at the entrance:
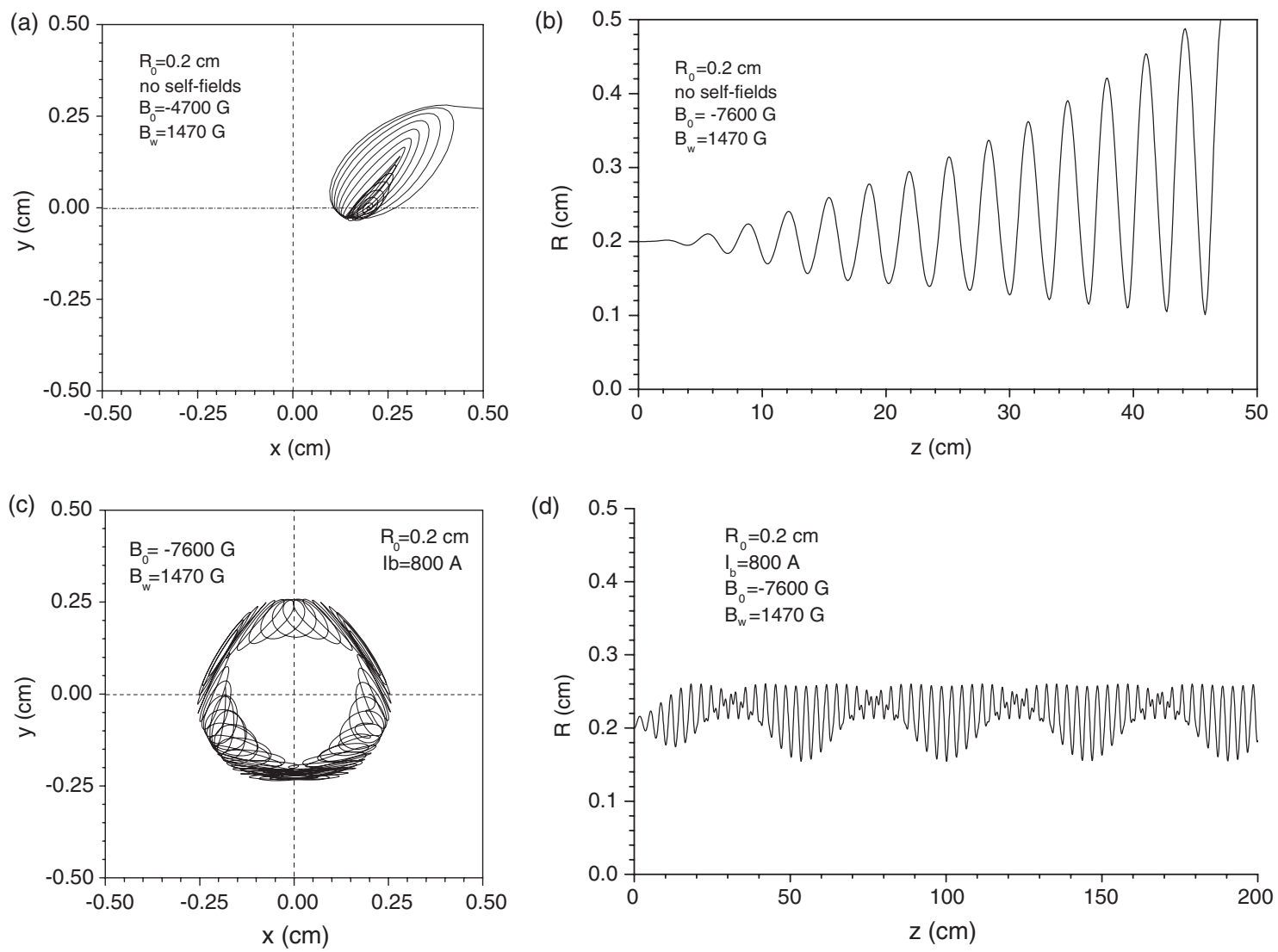

FIG. 5. Effect of the beam self-field on the electron motion: (a) and (b) without, and (c) and (d) with the self-field of the electron beam $\left(I_{b}=800 \mathrm{~A}\right)$ taken into account, where the other parameters are the same as in Fig. 1. 


$$
T=\frac{I_{b, \text { exit }}}{I_{b, \text { entrance }}}
$$

It is clear from the figure that indeed, the resonance occurs in a range of the positive guide magnetic field, and brings on loss of the total beam current, which is in agreement to both the analytical predication in the first paper of this series [25] and the experimental measurements [13].

Perhaps more interesting is at the antiresonance in a reversed guide magnetic field configuration reported by Conde and Bekefi, where only a fraction of beam current was lost in a range of the reversed guide magnetic field [see Fig. 3(b) in Ref. [13]]. The 3D wiggler harmonic approximation model $[14,15]$ could explain the beam current loss at the point of the reversed guide magnetic field $\left|\Omega_{0}\right|=$ $k_{w} v_{\| 0}$, but could not explain why it occurred in a range of the reversed guide magnetic field. Also, the so-called "2: -1 " version of the Cherry's model [29] failed to explain why the beam current loss was only a fraction in the experimental observations, but was not as significant a part as that model expected. Figure 4(b) plots our nonlinear simulation results of the beam transmission as a function of the reversed guide magnetic field. Principally, it is in agreement with the experimental observation and verifies the theoretical explanation in the first paper of this series [25].

We have noted that the self-field singularity model [30] imputed the beam loss at antiresonance to a new singularity generated by the self-field of the electron beam. However, the self-field plays a complex role in the equilibrium electron's motion, and on the contrary, it may be advantageous to confine the electron beam in certain parameters domain $[11,12,26]$. Below, we demonstrate by the nonlinear simulations that, at least for the parameters domain considered here, the self-field converges the electron's divergent trajectory. With the self-field being excluded, Figs. 5(a) and 5(b) plot the cross-sectional view and the radial position evolution along the axial position of an electron's trajectory at the antiresonance $\left(B_{0}=-7600 \mathrm{G}, B_{w}=\right.$ $1470 \mathrm{G}$ ), where the initial position $R_{0}=0.2 \mathrm{~cm}$ and other parameters are the same as in Fig. 1. It displays that the orbit diverges rapidly and the electron strikes the waveguide wall at the position $z=47 \mathrm{~cm}$. As shown in Figs. 5(c) and 5(d), however, dramatic change appears when the self-field of the electron beam with a current $I_{b}=800 \mathrm{~A}$ is taken into account: the orbit is confined well, the electron does not strike the waveguide wall and successfully passes through the wiggler. Focusing effect and modulation mechanism of the beam self-fields on the electron's motion is studied in a separate paper [26].

\section{CONCLUSIONS}

In this paper we have provided a nonlinear model to comprehensively simulate the equilibrium electrons' motion in a FEL with the combination of a threedimensional helical wiggler and a positive or reversed guide magnetic field, compared to the function of a positive guide magnetic field and a reversed guide magnetic field on the motion stability and beam transport, and clarified issues at resonance and at antiresonance. The following points can be concluded from the results: (1) A reversed guide magnetic field configuration brings the electron motion a much smaller Larmor radius and smaller transverse displacement span than a positive guide magnetic field does; consequently, the former provides a better transportation quality of the electron beam with smaller spreads and more perfect spot than the latter. (2) The electron motion far from the resonance and antiresonance is stable in both the positive and reversed guide magnetic field configurations. Nevertheless, fluctuation induced by a reversed guide magnetic field is smaller than that by a positive guide magnetic field, especially in the adiabatic region of the wiggler. (3) In a vicinal range of the resonance induced by a positive guide magnetic field, all the electrons of the beam are lost on the waveguide wall as the Larmor rotation rapidly tends to infinite, while in a vicinal range of the antiresonance induced by a reversed guide magnetic field, only a fraction of electrons are lost on the waveguide wall owing to the Larmor rotation conditionally tending finite. (4) In a certain parameters domain, the self-field of the electron beam is advantageous to confine the electrons' motion at antiresonance.

\section{ACKNOWLEDGMENTS}

This work was partly supported by the Youth Foundation of Sichuan Provincial Department of Education (Grant No. 10ZB080), the Foundation of Sichuan Provincial Department of Education (Grant No. 12ZB136), and the Xihua University Foundation (Grant No. Z1123329).

[1] V. L. Granatstein, S. P. Schlesinger, M. Herndon, R. K. Parker, and J. A. Pasour, Appl. Phys. Lett. 30, 384 (1977).

[2] D. B. McDermott, T. C. Marshall, S.P. Sprangle, R. K. Parker, and V.L. Granatstein, Phys. Rev. Lett. 41, 1368 (1978).

[3] T. Kwan and J. M. Dawson, Phys. Fluids 22, 1089 (1979).

[4] L. Friedland and J. L. Hirshfield, Phys. Rev. Lett. 44, 1456 (1980).

[5] P. Diament, Phys. Rev. A 23, 2537 (1981).

[6] H. P. Freund, S. Johnston, and P. Sprangle, IEEE J. Quantum Electron. 19, 322 (1983).

[7] S.-C. Zhang and Z. Zhang, Appl. Phys. Lett. 55, 1380 (1989).

[8] J. Fajans, D. A. Kirkpatrick, and G. Bekefi, Phys. Rev. A 32, 3448 (1985).

[9] C. Chen and R. C. Davidson, Phys. Fluids B 2, 171 (1990).

[10] C. Chen and R.C. Davidson, Phys. Rev. A 42, 5041 (1990).

[11] S.-C. Zhang and Y. Xu, Phys. Lett. A 179, 311 (1993). 
[12] S.-C. Zhang, Y. Xu, and Q. Liu, Phys. Rev. E 48, 3952 (1993).

[13] M.E. Conde and G. Bekefi, Phys. Rev. Lett. 67, 3082 (1991).

[14] K. R. Chu and A. T. Lin, Phys. Rev. Lett. 67, 3235 (1991).

[15] S.-C. Zhang and J. Elgin, Phys. Rev. E 55, 4684 (1997); also in Phys. Plasmas 11, 1663 (2004).

[16] N.S. Ginzburg, A. K. Kaminsky, A. A. Kaminsky, N. Y. Peskov, S. N. Sedykh, A. P. Sergeev, and A.S. Sergeev, IEEE Trans. Plasma Sci. 26, 536 (1998).

[17] A. V. Elahov, A. K. Kaminsky, E. A. Perelstein, S. N. Sedykh, and A. P. Sergeev, Phys. Rev. ST Accel. Beams 8, 090701 (2005).

[18] S. Khan, J. Mod. Opt. 55, 3469 (2008).

[19] V. Goryashko, K. Ilyenko, and A. Opanasenko, Phys. Rev. ST Accel. Beams 12, 100701 (2009).

[20] S. L. Sinitsky, A. V. Arzhannikov, V. T. Astrelin, P. V. Kalinin, and V.D. Stepanov, IEEE Trans. Plasma Sci. 37, 1885 (2009).
[21] A. K. Kaminsky, E. A. Perelshtein, S. N. Sedykh, N. S. Ginzburg, S. V. Kuzikov, N. Yu. Peskov, and A.S. Sergeev, Tech. Phys. Lett. 36, 211 (2010).

[22] V. Goryashko, Phys. Rev. ST Accel. Beams 14, 030703 (2011).

[23] N.S. Ginzburg, I. I. Golubev, A. K. Kaminsky, A.P. Kozlov, E. A. Perelshtein et al., Phys. Rev. ST Accel. Beams 14, 041002 (2011).

[24] J. Fajans, G. Bekefi, Y.Z. Yin, and B. Lax, Phys. Fluids 28, 1995 (1985).

[25] Shi-Chang Zhang, Xiao-Li Huang, Shi-Jian Wang, and Yong-Gen $\mathrm{Xu}$ (unpublished).

[26] Shi-Chang Zhang, Phys. Lett. A 377, 319 (2013).

[27] H. P. Freund and A. K. Ganguly, Phys. Rev. A 33, 1060 (1986).

[28] G. Benettin, L. Galgani, and J.-M. Strelcyn, Phys. Rev. A 14, 2338 (1976).

[29] J. T. Donohue and J. L. Rullier, Nucl. Instrum. Methods Phys. Res., Sect. A 475, 137 (2001).

[30] K. H. Tsui, Phys. Plasmas 2, 3865 (1995). 